\title{
TRANSCENDENTAL NUMBERS.
}

An authorized translation by Professor W. W. BeMan of Chapter XXV. of Vol. II., of the Lehrbuch der Algebra*

BY PROFESSOR HEINRICH WEBER.

[Professor Weber's presentation of the recent methods of demonstrating the transcendency of $e$ and $\pi$, especially in sections 205 and 206 , is so elementary that its reproduction in English will be welcomed by many. For the sake of completeness the whole chapter has been given. W. W. B.]

$\S 203$.

\section{Enumerable Masses.}

In the introduction to our work the general number concept was defined and with this general number concept we have operated, e.g., in the proof of the existence of a root. In the further course of our investigations, we have dealt only with algebraic numbers without stopping to inquire whether the content of the number domain was thus exhausted, or there were non-algebraic numbers besides. The existence of non-algebraic numbers, also called transcendental, was first demonstrated by Liouville. Other proofs have been devised by G. Cantor. $\dagger$

We begin here with the conception, already established in the introduction, of a mass or manifoldness, which means a system of elements of any kind so far defined that in case of any arbitrary object it is possible to decide whether it belongs to the system or not.

We distinguish between finite and infinite masses and introduce as our first and most important example of an infinite mass the aggregate of the natural numbers $1,2,3, \ldots$. The following definition then holds :

1. Definition. A mass is said to be enumerable [abzählbar] when its elements can be brought into a $(1,1)$ correspondence with the whole series of natural numbers or a portion of the same.\$

Accordingly every finite mass is enumerable and in the enumeration the series of natural numbers is used only up to a certain greatest number. In what follows we specially consider infinite masses.

* Braunschweig, Vieweg und Sohn, 1895 und 189ô.

$\dagger$ G. CANTor, Crelle's Journal, vol. 77 (1873). "Ueber eine Eigenschaft des Inbegriffes aller reellen algebraischen Zahlen."

$\mp$ CANTOR, $l$. $c$. The notion of enumerable masses agrees with the notion of simply infinite systems defined by DEDEKIND without the assumption of the number system. DEDEKIND, "Was sind und was sollen die Zahlen?" \& 6. Braunschweig, 1887. (Second unaltered edition, 1893.) 
We may also define an infinite enumerable mass as a mass of such a nature that to every element a definite number from the series of natural numbers can be applied as a name, provided every number of the series of natural numbers is employed ; that is, a mass in which there is a first, second, third,... . hundredth . . . element.

Finally, we may also say that an infinite enumerable mass is one whose elements can be arranged in such an order that there is a first element, that each element is followed by a definite other one of the mass, and that every element save the first is preceded by a definite other element. Such a series we may call a countable [zählbar] arrangement. It is clear that an enumerable mass is enumerable not only in one way, but in infinitely many different ways.

Besides the series of natural numbers which is manifestly enumerable, we may mention as a second example the system of rational positive proper fractions which may be enumerated, for instance, in the following manner:

$$
\frac{1}{2}, \frac{1}{3}, \frac{2}{3}, \frac{1}{4}, \frac{3}{4}, \frac{1}{5}, \frac{2}{5}, \frac{3}{5}, \frac{4}{5}, \frac{1}{6}, \frac{5}{6}, \ldots
$$

$i . e$, so that every greater denominator follows the less denominator, and that the fractions with equal denominators are arranged according to the magnitude of the numerators. If, however, the fractions were to be arranged in order of numerical value, we should not have a countable arrangement.

2. The aggregate of all algebraic numbers is an enumerable mass.

To prove this important theorem we recall that every algebraic number $\theta$ is the root of one and only one irreducible equation

$$
f(\theta)=a_{0} \theta^{n}+a_{1} \theta^{n-1}+\ldots+a_{n}=0
$$

where $a_{0}, a_{1}, \ldots a_{n}$ are integral rational numbers without a common divisor, and $a_{0}$ is positive and different from zero. The degree $n$ of the equation (1) is a positive integer and hence at least equal to 1 . Some of the numbers $a_{1}, a_{2}, \ldots a_{n-1}$ may be 0 .

We will now select the signs \pm so that $\pm a_{1}, \pm a_{2}, \ldots \pm a_{n}$ are not negative, and call the sum

$$
N=(n-1)+a_{0} \pm a_{1} \pm a_{2} \ldots \pm a_{n}
$$

the height of the algebraic number $\theta$. The height is then always a positive integer.

Now it is easy to see that for a given value of the height 
$N$ there is always only a finite number of algebraic numbers. For in the first place by (2) $n$ can never be greater than $N$, and for given values of $N$ and $n$ the numbers $a_{1}, a_{2}, \ldots a_{n}$ can be determined in only a finite number of ways. Among the functions $f(\theta)$ so determined we retain only the irreducible ones. If now the algebraic numbers be arranged so that the numbers of less height precede those of greater height, that among numbers of equal height that one precedes whose real part is the smaller, that among numbers of equal height and equal real part the one of smaller imaginary part precedes, we have a countable arrangement of the algebraic numbers and it is shown that the aggregate of all algebraic numbers is an enumerable mass.

We have, for example:

$$
\begin{aligned}
& N=1, n=1, a_{0}=1, a_{1}=0, \\
& N=2, n=1, a_{0}=1, a_{1}= \pm 1 \text {, } \\
& N=3, n=1, a_{0}=1, a_{1}= \pm 2 \text {, } \\
& a_{0}=2, a_{1}= \pm 1 \text {, } \\
& n=2, a_{0}=1, a_{1}=0, a_{2}=1 \text {, }
\end{aligned}
$$

and the beginning of an orderly series of algebraic numbers becomes

$$
0,-1,+1,-2,-\frac{1}{2},-\sqrt{-1}, \sqrt{-1}, \frac{1}{2}, 2, \ldots
$$

Every portion of the series of natural numbers is an enumerable mass : for we have only to arrange the numbers of such portion in order of magnitude to obtain a countable arrangement.

Thus it appears that every portion of an enumerable mass is itself an enumerable mass. Hence it follows among other things that the mass of real algebraic numbers is enumerable.

$$
\S 204 .
$$

Unenumerable Masses.

We now proceed to the proof of the theorem that there are number masses which are not enumerable and in particular we shall show that :

The aggregate of all real numbers even when we are restricted to a finite interval is not enumerable.

For this purpose consider any enumerable mass of unequal real numbers which in a countable arrangement $(\Omega)$ may be designated: 


$$
\omega_{1}, \omega_{2}, \omega_{3}, \omega_{4}, \ldots
$$

(where it is to be observed that the elements may not be arranged in order of magnitude).

For brevity we shall speak of that one of two elements of the series $\Omega$ which has the smaller index as the earlier, the one with greater index as the later.

We now select any two real numbers $\alpha$ and $\beta, \alpha<\beta$, and show that in the interval $\delta=(\alpha, \beta)$ there is at least one number not found in the series $\Omega$. As soon as we have shown the existence of one such number for every interval, there must be infinitely many, since we can apply the same argument to every portion of the interval $(\alpha, \beta)$.

First, it is clear that our proposition is correct if only a finite number of numbers of the series $\Omega$ is contained in any finite portion of the interval $\delta$, and we may, therefore, pass immediately to the case where an infinite mass of numbers of the series $\Omega$ lies in every part, no matter how small, of the interval $\delta$.

Designate by $\alpha_{1}, \beta_{1}$ the two earliest numbers of the series $\Omega$, which lie in the interval $\delta$, assume $\alpha_{1}<\beta_{1}$ and put $\delta_{1}=\beta_{1}-\alpha_{1}$, so that $\delta_{1}=\left(\alpha_{1}, \beta_{1}\right)$ is a portion of the interval $\delta$.

Now designate similarly by $\alpha_{2}, \beta_{2}$ the two earliest numbers of the series $\Omega$ which lie within the interval $\delta$, (exclusive of the limits), assume $\alpha_{2}<\beta_{2}$ and put $\delta_{2}=\beta_{2}-\alpha_{2}$.

We can proceed in this way and obtain an unlimited series of intervals

$$
\delta, \delta_{1}, \delta_{2}, \delta_{3}, \ldots,
$$

each of which includes all that follow, and two series of numbers :

$$
\begin{aligned}
& \alpha, \alpha_{1}, \alpha_{2}, \ldots, \\
& \beta, \beta_{1}, \beta_{2}, \ldots,
\end{aligned}
$$

all of which, with the possible exception of the first $\alpha, \beta$, belong to the series $\Omega$. The numbers $\alpha, \alpha_{1}, \alpha_{2}, \ldots$, form an increasing series, the numbers $\beta, \beta_{1}, \beta_{2}, \ldots$, a decreasing series, and at the same time every $\alpha$ is less than every $\beta$.

1. Hence it follows that the numbers $\alpha_{\nu}$ have an upper limit $a$, the numbers $\beta_{\nu}$ a lower limit $b$ and that $a \leqq b$ (see Vol. I., § 38, 1.) Possibly we may have $a=b$.

From the method of formation of the intervals $\delta, \delta_{1}, \delta_{2}, \ldots$ it follows that:

2. If any number $\omega$ of the series $\Omega$ falls in the interval $\delta_{\nu}$, excluding its limits $\alpha_{\nu}, \beta_{\nu}$, then $\omega$ occurs later in the series $\Omega$ than the pair of numbers $\alpha_{\nu}, \beta_{\nu}$, belonging to the same series. 
For $\alpha_{\nu}, \beta_{\nu}$ were the two earliest numbers of the series $\Omega$ occurring in the interval $\delta_{\nu-1}$. Hence it follows that:

3. The greater the index $\nu$, the later are the two numbers $\alpha_{\nu}, \beta_{\nu}$ in the series $\Omega$, and as we have assumed the series of intervals $\delta_{\nu}$ not to terminate; we can advance $\alpha_{\nu}, \beta_{\nu}$ as far as we please in the series $\Omega$ by increasing $\nu$ sufficiently.

Now it follows very readily that no number $g$ which coincides with one of the numbers $a, b$, or, if $a$ and $b$ are unequal, lies between them, can belong to the series $\Omega$.

For the number $g$ lies within each one of the intervals $\delta_{\nu}$. Now if we assume that $g$ occurs in $\Omega$, and by (3) increase $\nu$ so that $\alpha_{\nu}, \beta_{\nu}$ come later in $\Omega$ than $g$, then by (2) $g$ cannot lie in the interval $\delta_{\nu}$ and thus the absurdity of our hypothesis is demonstrated.

Hence it follows that the aggregate of all the numbers of an interval $\alpha, \beta$ does not form an enumerable mass.

This theorem may be demonstrated in another way which is simpler in some respects and may be briefly indicated. We do not restrict the generality if we confine ourselves to the interval from 0 to 1 . We shall imagine all numbers of this interval represented by decimal fractions with an infinite number of terms. Finite decimal fractions are included if we make all the digits after a certain one equal to zero. To render this representation by decimal fractions unambiguous, it must be agreed that for a finite decimal fraction this representation must always be chosen, so that, for example, $0.4999 \ldots$ must not be written for $0.5000 \ldots$

We will now assume that these decimal fractions form an enumerable mass. They may then be arranged in a countable series, represented as follows:

$$
\begin{gathered}
\omega_{1}=0 . \alpha_{1}^{(1)} \alpha_{2}^{(1)} \alpha_{3}^{(1)} \ldots \\
\omega_{2}=0 . \alpha_{1}^{(2)} \alpha_{2}^{(2)} \alpha_{3}^{(2)} \ldots \\
\omega_{3}=0 . \alpha_{1}^{(3)} \alpha_{2}^{(3)} \alpha_{3}^{(3)} \ldots \\
\ldots \ldots \ldots \ldots \ldots \ldots \ldots
\end{gathered}
$$

where the $\alpha_{\mu}^{(\nu)}$ represent digits of the decimal system.

But it is very easy to form a decimal fraction (or indeed, as many as we please) which is not contained in the series $\Omega$. We have only to form

$$
\eta=0 . \beta_{1} \beta_{2} \beta_{3} \ldots
$$

where the $\beta_{\nu}$ are digits of the decimal system, satisfying the one condition that for every $\nu, \beta_{\nu}$ is different from $\alpha_{\nu}^{(\nu)}$. This 
number $\eta$, which also belongs to the interval $(0,1)$ cannot be a number of the series $\Omega$.

The formation of $\eta$ may be made still more general by arbitrarily selecting the $\beta$ 's as far as we please and then applying the law, $\beta_{\nu} \gtreqless \alpha_{\nu}^{(\nu)}$.

Since it has thus been shown that the real algebraic numbers form an enumerable mass and that in every interval there are numbers which do not belong to a given enumerable mass it follows immediately that:

In every real interval there are transcendental numbers.

$$
\S 205 .
$$

Transcendency of the Number $e$.

It is a far more difficult task to decide whether any given number is algebraic or transcendental. Here interest has chiefly been concentrated upon the two numbers which occur so frequently in analysis, $e$, the base of the natural system of logarithms and the Ludolphian number $\pi$, the ratio of the circumference of the circle to the diameter.

For the number $e$ the question was answered by Hermite in a celebrated memoir, $*$ which has been made the basis of the later investigations upon the number $\pi$. The solution for the number $\pi$, which was specially interesting on account of its relation to the famous problem of the quadrature of the circle, for a long time, however, presented insurmountable difficulties. Finally, Lindemann succeeded in demonstrating that $\pi$ also belongs to the transcendental numbers. But Lindemann's proof still presented great difficulties. These, however, have gradually been so completely removed by the investigations of Weierstrass, Hilbert, Hurwitz and Gordan $†$ that the demonstration can now be made with quite elementary means and in the most simple manner.

If we represent by $\Pi(n)$ the product

$$
\Pi(n)=1 \cdot 2 \cdot 3 \ldots n
$$

then, for any $x$,

* Hermite, "Sur la function exponentielle." Comptes rendus, vol. LXXVII, 1873.

† LiNDEMANN, "Ueber die Zahl $\pi . "$ Mathem. Annalen, vol. 20, 1882. WEIERSTRASS, "Zu Lindemann's Abhandlung über die Ludolph'sche Zahl." Sitzungsbericht der Berliner Akademie, December 3, 1885. The articles by HILBERT, HURWITZ and GoRDAN are all three found in vol. 43, Mathem. Annalen (1893), the first two also in the Göttinger Nachrichten of 1893. 


$$
\frac{x^{n}}{I I(n)}
$$

as $n$ increases indefinitely, approaches the limit zero and that more rapidly than the terms of a decreasing geometrical progression. For if $k$ is an integer $>|x|$ then is $\left|\frac{x}{h}\right|$ always a proper fraction if $h \geqq k$. Consequently

$$
\left.\frac{x^{n}}{\Pi(n)}|=| \frac{x^{k}}{\Pi(k)} \frac{x}{k+1} \frac{x}{k+2} \cdots \frac{x}{n}|<| \frac{x^{k}}{\Pi(k)}|| \frac{x}{k}\right|^{n-k} .
$$

Hence it follows that the infinite series

$$
e^{x}=1+x+\frac{x^{2}}{\Pi(2)}+\frac{x^{3}}{\Pi(3)}+\ldots
$$

converges for all values of $x$, and by it we define the exponential function $e^{x}$, whose simplest properties we here assume as known from analysis. We have, in particular, the relation

$$
e^{x+y}=e^{x} e^{y}
$$

for any two values $x, y$; whence it follows that, for $n$ a positive integer, $e^{n}$ is the $n^{\text {th }}$ power of the number

$$
\text { (3) } e=2+\frac{1}{\Pi(2)}+\frac{1}{I l(3)}+\ldots=2.718281828459 \ldots
$$

If now $r$ represents any positive integer, formula (2) may be written

(4) $\Pi(r) e^{x}=\Pi(r)+\frac{\Pi(r)}{\Pi(1)} x+\frac{\Pi(r)}{\Pi(2)} x^{2}+\ldots+x^{r}+x^{r} U_{r}$, where

(5) $U_{r}=\frac{x}{r+1}+\frac{x^{2}}{(r+1)(r+2)}+\frac{x^{3}}{(r+1)(r+2)(r+3)}+\ldots$

and this formula holds for $r=0$ if we assume $I(0)=1$.

Designating $|x|$ by $\xi$, it follows from the theorem that the absolute value of a sum is never greater than the sum of the absolute values of its parts, that

$$
\left|U_{r}\right| \leqq \frac{\xi}{r+1}+\frac{\xi^{2}}{(r+1)(r+2)}+\frac{\xi^{3}}{(r+1)(r+2)(r+3)}+\ldots
$$

and therefore 
If we put

$$
\left|U_{r}\right|<\mid 1+\frac{\xi}{1}+\frac{\xi^{2}}{1 \cdot 2}+\frac{\xi^{3}}{1 \cdot 2 \cdot 3}+\ldots=e^{\xi} .
$$

$$
U_{r}=q_{r} e^{\xi}
$$

then $q_{r}$ is a function of $x$ of which we know that $\left|q_{r}\right|<1$.

We now substitute in formula $(4) r=0,1, \ldots n$, and write out the terms in reverse order as follows:

$$
\begin{gathered}
\Pi(n) e^{x}=q_{n} x^{n} e^{\xi}+x^{n}+n x^{n-1}+n(n-1) x^{n-2}+ \\
\ldots+\Pi(n)
\end{gathered}
$$

$$
\begin{gathered}
I I(n-1) e^{x}=q_{n-1} x^{n-1} e^{\xi}+x^{n-1}+(n-1) x^{n-2} \\
\quad+(n-1)(n-2) x^{n-3}+\ldots \\
\ldots \ldots \ldots \ldots \\
e^{x}=q_{0} e^{\xi}+1 .
\end{gathered}
$$

Now assume an arbitrary integral function of $x$ of the $n^{\text {th }}$ degree :

$$
f(x)=c_{n} x^{n}+c_{n-1} x^{n-1}+c_{n-2} x^{n-2}+\ldots+c_{0},
$$

and its derivatives

$$
\begin{aligned}
& f^{\prime}(x)=n c_{n} x^{n-1}+(n-1) c_{n-1} x^{n-2}+\ldots \\
\text { (8) } f^{\prime \prime}(x) & =n(n-1) c_{n} x^{n-2}+(n-1)(n-2) c_{n-1} x^{n-8}+\ldots \\
& \ldots \ldots \ldots \ldots \ldots \\
& f^{(n)} x=I I(n) c_{n}
\end{aligned}
$$

and for brevity put

$$
F(x)=f(x)+f^{\prime}(x)+f^{\prime \prime}(x)+\ldots+f^{(n)}(x) .
$$

Then $F(x)$ is an integral function of $x$ of degree $n$. Finally assume

$$
\begin{aligned}
Q(x) & =c_{n} q_{n} x^{n}+c_{n-1} q_{n-1} x^{n-1}+\ldots+c_{0} q_{0}, \\
P & =c_{n} I I(n)+c_{n-1} I(n-1)+\ldots+c_{0} .
\end{aligned}
$$

$Q(x)$ depends upon $x$, though not expressible rationally in terms of $x ; P$, however, is independent of $x$.

If we now multiply equations (6) in order by $c_{n}, c_{n-1}, \ldots c_{0}$ and add, we get

$$
e^{x} P=F(x)+e^{\xi} Q(x) .
$$


This formula will serve as the foundation for the following deductions.

If we assume $e$ to be an algebraic number it must satisfy an equation of degree $m$ (say)

$$
C_{0}+C_{1} e+C_{2} e^{2}+\ldots+C_{m} e^{m}=0
$$

whose coefficients $C_{0}, C_{1}, C_{2}, \ldots C_{m}$ are integral rational numbers, * and $C_{0}$ and $C_{m}$ are not zero. It is required to show the absurdity of this hypothesis.

To this end we substitute in (12) for $x$ successively the integers $0,1,2, \ldots m$, so that $\xi$ becomes identical with $x$, multiply by $C_{0}, C_{1}, \ldots C_{m}$ and add.

Then by (13), since $P^{m}$ is independent of $x$,

$$
\begin{aligned}
0 & =C_{0} F(0)+C_{1} F(1)+\ldots+C_{m} F(m) \\
& +C_{0} Q(0)+C_{1} e Q(1)+\ldots+C_{m} e^{m} Q(m)
\end{aligned}
$$

and now by a suitable choice of the still arbitrary function $F(x)$ we are to show that (14) is impossible.

We select a prime number $p$, which is greater than $m, \dagger$ and put

$$
F(x)=\frac{x^{p-1}(1-x)^{p}(2-x)^{p} \cdots(m-x)^{p}}{11(p-1)}
$$

so that the degree $n$ of $f(x)$ is equal to $(m+1) p-1$ and we now prove two things :

(1) $C_{0} F(0)+C_{1} F(1)+\ldots+C_{m} F(m)$ is an integer different from zero and, therefore, disregarding sign, at least equal to 1 .

(2) $C_{0} Q(0)+C_{1} e Q(1)+\ldots+C_{m} e^{m} Q(m)$ is less than 1, both upon the assumption that $p$ has been suitably disposed of. If these two points are established, we recognize

* The following definitions are given by Weber in 8133 :

An algebraic number $\theta$ is aalled an integral algebraic number when it satisfies a rational equation

$$
\Theta^{m}+A_{1} \theta^{m-1}+\ldots+A_{m-1} \Theta+A_{m}=0
$$

whose coefficients $A_{1}, A_{2}, \ldots, A_{m}$ are integers.

Integral algebraic numbers include as a special case ordinary integers, which, for distinction, we call integral rational numbers.

W. W. B.

† That there is always a prime number $p$ greater than any given num ber $\mu$ was already demonstrated in EucLID (Elements, Book IX., Prop. XX., vol. 2 of HeIBERG's edition). The proof is simply this, that the integer $\Pi(\mu)+1$ which is obviously greater than $\mu$ is divisible by no prime number, which is not greater than $\mu$, because this number gives the remainder 1 when divided by the numbers $2,3, \ldots \mu$. Hence it is impossible that there be no prime number above $\mu$. 
the impossibility of equation (14) and therefore of equation (13), from which (14) was derived.

If we arrange the numerator of $f(x)$ in powers of $x$ we get an expression of the form :

$$
f(x)=\frac{A_{p-1} x^{p-1}+A_{p} x^{p}+A_{p+1} x^{p+1}+\ldots}{\Pi(p-1)},
$$

where $A_{p-1}, A_{p}, A_{p+1}, \ldots$ are integers and $A_{p-1}=[\Pi(m)]^{p}$, and hence is certainly not divisible by $p$. Comparing (16) with Taylor's Formula

we have

$$
f(x)=f(0)+x f^{\prime}(0)+\frac{x^{2}}{I I(2)} f^{\prime \prime}(0)+\ldots
$$

$$
\begin{gathered}
f(0)=f^{\prime}(0)=f^{\prime \prime}(0)=\ldots=f^{(p-2)}(0)=0 \\
f^{(p-1)}(0)=A_{p-1}, f^{(p)}(0)=p A^{p}, f^{(p+1)}(0)=p(p+1) A_{p+1}, \ldots
\end{gathered}
$$

Hence

$$
F(0)=A_{p-1}+p A_{p}+p(p+1) A_{p+1}+\ldots
$$

is an integer not divisible by $p$. If, however, we arrange $f(x)$ in powers of $(x-1)$, we get

$$
f(x)=\frac{B_{p}(x-1)^{p}+B_{p+1}(x-1)^{p+1}+\ldots}{\Pi(p-1)},
$$

where $B_{p}, B_{p+1}, \ldots$ are again integers. Thence it follows as above by comparison with

that

$$
f(x)=f(1)+(x-1) f^{\prime}(1)+\frac{(x-1)^{2}}{\Pi(2)} f^{\prime \prime}(1)+\ldots
$$

$$
F(1)=p B_{p}+p(p+1) B_{p+1}+\ldots
$$

Consequently, $F(1)$ is an integer divisible by $p$, and similarly we can show that $F(2), F(3), \ldots F(m)$ are integers divisible by $p$. Since $p$ can be chosen so large that $C_{0}$ is not divisible by $p$, it follows that

$$
C_{0} F(0)+C_{1} F(1)+\ldots+C_{m} F(m)
$$

is an integer not divisible by $p$ and hence does not vanish; thus (1) is demonstrated.

If we can show further that by increasing $p, Q(x)$ can for any positive $x$ be made as small as we please, it will follow that for a sufficiently large value of $p$ the expression (2) can certainly be made less than 1 , and our proof is completed. 
Returning to the expression (10) for $Q(x)$, let $\gamma_{n}, \gamma_{n-1}$, $\ldots \gamma_{0}$ denote the absolute values of the coefficients $c_{n}, c_{n-1}, \ldots c_{0}$ of $f(x)$. Since $q_{n}, q_{n-1}, \ldots$ are less in absolute value than 1 , it appears that for every positive $x,|Q(x)|$ is less than

$$
\psi(x)=\gamma_{n} x^{n}+\gamma_{n-1} x^{n-1}+\ldots+\gamma_{0} .
$$

The coefficients $c_{n}, c_{n-1}, \ldots c_{0}$ of the function $f(x)$ in (15) differ from the $\gamma_{n}, \gamma_{n-1}, \ldots \gamma_{0}$ only in sign. If we replace $x$ by $-x$, thus forming the function

$$
f(-x)=\frac{x^{p-1}(1+x)^{p}(2+x)^{p} \ldots(m+x)^{p}}{\Pi(p-1)},
$$

this function has the same coefficients as $f(x)$ but with all the signs positive. It is therefore nothing else than the function $\psi(x)$.

If we put

then

$$
X=x(1+x)(2+x) \ldots(m+x),
$$

$$
\psi(x)=\frac{X}{x} \cdot \frac{X^{p-1}}{I I(p-1)}
$$

and, as shown at the beginning of this section, with $p$ increasing indefinitely this approaches the limit zero.

Therefore $e$ is a transcendental number.

$$
\S 206 .
$$

Transcendency of the number $\pi$.

By the same methods we may now show the transcendency of the number $\pi$. As definition of this number we use the property that it is the least positive number which satisfies the equation

$$
e^{i \pi}=-1,
$$

when $e^{x}$ is defined by the formula $\S 205$ (2).

If we assume now that $\pi$ and consequently $i \pi$ also is an algebraic number, then is $i \pi$ one of the roots of an irreducible rational equation $\chi(x)=0$ whose coefficients are integral rational numbers.

If we denote the various roots of this algebraic equation by $\beta_{1}, \beta_{2}, \ldots \beta_{\nu}$ and the coefficient of $x^{\nu}$ in $\chi$ by $\alpha$, then

$$
\chi(x)=a\left(x-\beta_{1}\right)\left(x-\beta_{2}\right) \ldots\left(x-\beta_{v}\right),
$$

and the products $a \beta_{1}, a \beta_{2}, \ldots \alpha \beta_{\nu}$ are integral algebraic num- 
bers and their integral symmetric functions therefore integral rational numbers ( $\$ 133$ ).

As the number $i \pi$ must occur among the $\beta$ 's, we must have by (1):

$$
\left(1+e^{\beta_{1}}\right)\left(1+e^{\beta_{2}}\right) \ldots\left(1+e^{\beta_{v}}\right)=0,
$$

or expanding

$$
1+\sum e^{\beta_{i}}+\sum e^{\beta_{i}+\beta_{h}}+\sum e^{\beta_{i}+\beta_{h}+\beta_{k}}+\cdots=0 .
$$

Among the exponents in this sum the number 0 may occur several times; suppose $(C-1)$ times so that $C$ is a positive integer at least $=1$. The remaining exponents $\beta_{i}, \beta_{i}+\beta_{h}, \beta_{i}+\beta_{h}+\beta_{k}, \ldots$, some of which may be equal to one another, we will designate by $\alpha_{1}, \alpha_{2}, \ldots \alpha_{\mu}$, so that the equation becomes.

$$
C+e^{a_{1}}+e^{a_{2}}+\ldots+e^{a_{\mu}}=0 .
$$

The quantities $\alpha_{1}, \alpha_{2}, \ldots \alpha_{\mu}$ are algebraic numbers which multiplied by the rational integer $a$ become integral algebraic numbers. The symmetric functions of the various sums $\beta_{i}, \beta_{i}+\beta_{h}, \beta_{i}+\beta_{h}+\beta_{k}, \ldots$ are at the same time symmetric functions of $\beta_{1}, \ldots \beta_{\nu}$, and therefore rational numbers. These sums are therefore the roots of a rational equation and since the root 0 can be removed as often as it occurs, the quantities $\alpha_{1}, \alpha_{2}, \ldots \alpha_{\mu}$ are also roots of a rational equation. The fundamental symmetric functions of the quantities $a \alpha_{1}, a \alpha_{2}, \ldots a \alpha_{\mu}$ are integral rational numbers.

The absolute values of the numbers

$$
\alpha_{1}, \alpha_{2}, \ldots \alpha_{\mu}
$$

we shall designate by

$$
a_{1}, a_{2}, \ldots a_{\mu} \text {. }
$$

If in equation (12) of the previous section we put $x=0, \alpha_{1}, \alpha_{2}, \ldots \alpha_{\mu}$, and apply equation (3), we obtain

$$
\text { (4) } \begin{aligned}
0 & =C F(0)+F\left(\alpha_{1}\right)+F\left(\alpha_{2}\right)+\ldots+F\left(\alpha_{\mu}\right) \\
& +C Q(0)+e^{\alpha_{1}} Q\left(\alpha_{1}\right)+e^{\alpha_{2}} Q\left(\alpha_{2}\right)+\ldots+e^{\alpha_{\mu}} Q\left(\alpha_{\mu}\right),
\end{aligned}
$$

and we have to show the impossibility of this equation by a suitable choice of $f(x)$.

Let $p$ again be a prime number which may increase without limit, and put 


$$
f(x)=\frac{\alpha^{\mu p+p-1} x^{p-1}\left(x-\alpha_{1}\right)^{p}\left(x-\alpha_{2}\right)^{p} \ldots\left(x-\alpha_{\mu}^{-}\right)^{p}}{\Pi(p-1)}
$$

so that $f(x)$ is a function with rational coefficients.

As in the previous section we arrange in powers of $x$ :

$$
f(x)=\frac{A_{p-1} x^{p-1}+A_{p} x^{p}+A_{p+1} x^{p+1}+\ldots}{\Pi(p-1)}
$$

where $A_{p-1}, A_{p}, A_{p+1}, \ldots$ are integral rational numbers and

$$
A_{p-1}=(-1)^{\mu} \alpha^{\mu p+p-1} \alpha_{1}^{p} \alpha_{2}^{p} \ldots \alpha_{\mu}^{p} .
$$

If we assume $p$ greater than either of the rational integers

$$
a, a^{\mu} \alpha_{1} \alpha_{2} \ldots \alpha_{\mu},
$$

$A_{p-1}$ will not be divisible by $p$ and hence

$$
F(0)=A_{p-1}+p A_{p}+p(p+1) A_{p+1}+\ldots
$$

will be an integer not divisible by $p$. Likewise if $p$ is taken sufficiently large $C$ is not divisible by $p$.

On the other hand, arranging the numerator of $f(x)$ in powers of $\alpha\left(x-\alpha_{1}\right)$ we obtain

$$
f(x)=\frac{B_{p} a^{p}\left(x-\alpha_{1}\right)^{p}+B_{p+1} a^{p+1}\left(x-\alpha_{1}\right)^{p+1}+\ldots,}{I(p-1)},
$$

where $B_{p}, B_{p+1}, \ldots$ are no longer rational but are integral algebraic numbers, since the numerator of $f(x)$ is an integral function of $\alpha x, a \alpha_{1}, \ldots \alpha \alpha_{\mu}$.

From this it follows as in the last section that

$$
F\left(\alpha_{1}\right)=p B_{p} a^{p}+p(p+1) B_{p+1} a^{p+1}+\ldots
$$

If similarly we form $F\left(\alpha_{2}\right), F\left(\alpha_{3}\right), \ldots F\left(\alpha_{\mu}\right)$ and observe that the sum

$$
B_{p}\left(\alpha_{1}\right)+B_{p}\left(\alpha_{2}\right)+\ldots+B_{p}\left(\alpha_{\mu}\right)
$$

is an integral rational number, it follows that

$$
F\left(\alpha_{1}\right)+F\left(\alpha_{2}\right)+\ldots+F\left(\alpha_{\mu}\right)
$$

is an integral rational number divisible by $p$. Hence finally we conclude that

$$
C F(0)+F\left(\alpha_{1}\right)+F\left(\alpha_{2}\right)+\ldots+F\left(\alpha_{\mu}\right)
$$

is an integral rational number not divisible by $p$, and hence not equal to zero; it must therefore, disregarding sign, be at least equal to 1 . 
If now finally it can be shown that with the present choice of $f(x)$ the function $Q(x)$ can be made as small as we please, for any value of $x$, by a suitable increase of $p$, we may show, as before, the impossibility of equation (4).

This may be seen as follows: Instead of the function

$$
f(x)=c_{n} x^{n}+c_{n-1} x^{n-1}+\ldots+c_{0}
$$

consider the function

$$
\begin{aligned}
\psi(x) & =\frac{a^{\mu p+p-1} x^{p-1}\left(x+a_{1}\right)^{p}\left(x+a_{2}\right)^{p} \ldots\left(x+a_{\mu}\right)^{p}}{\Pi(p-1)} \\
& =\gamma_{n} x^{n}+\gamma_{n-1} x^{n-1}+\ldots+\gamma_{0},
\end{aligned}
$$

which has all its coefficients positive, (though not necessarily rational). The coefficients $c_{n}, c_{n-1}, \ldots$ are formed by multiplication and addition from the numbers $a,-\alpha_{1},-\alpha_{2}$, $\ldots-\alpha_{\mu}$, and the corresponding coefficients $\gamma_{n}, \gamma_{n-1}, \ldots$ are obtained from these on replacing $-\alpha_{1},-\alpha_{2}, \ldots-\alpha_{\mu}$ by their absolute values $a_{1}, a_{2}, \ldots a_{\mu}$; whence by the theorem previously mentioned it follows that the coefficients $\gamma_{n}, \gamma_{n-1}$, ... are certainly not less than the absolute values of the corresponding coefficients $c_{n}, c_{n-1}, \ldots$

Now for every finite $x$ whose absolute value is $\xi$, the absolute value of $Q(x)$ by $\$ 205(10)$ is not greater than

$$
\gamma_{n} \xi^{n}+\gamma_{n-1} \xi^{n-1}+\ldots+\gamma_{0}=\psi(\xi),
$$

and that $\psi(\xi)$ can be made as small as we please by increasing $p$ sufficiently appears from the form

where

$$
\psi(x)=\frac{X}{a x} \cdot \frac{X^{p-1}}{\Pi(p-1)},
$$

$$
X=a^{\mu+1} x\left(x+a_{1}\right)\left(x+a_{2}\right) \ldots\left(x+a_{\mu}\right) .
$$

Hence it is proved that:

The number $\pi$ is a transcendental number. The quadrature of the circle can not be effected by any geometric construction in which only algebraic curves and surfaces are employed.

$$
\S 207 .
$$

\section{Lindemann's General Theorem Regarding the Exponential} Function.

The transcendency of the numbers $e$ and $\pi$, just demonstrated, is contained as a special case in a very general theorem regarding the exponential function announced by 
Lindemann in the memoir above mentioned, of which an extended proof was given by Weierstrass in his memoir. We propose to demonstrate this theorem here by the use of the same methods as in the two special cases. The theorem may be stated as follows:

I. An equation of the form

$$
C_{1} e^{z_{1}}+C_{2} e^{z_{2}}+\ldots+C_{m} e^{z_{m}}=0
$$

is impossible if the coefficients $C_{1}, C_{2}, \ldots C_{m}$ are algebraic numbers and the exponents $z_{1}, z_{2}, \ldots z_{m}$ are distinct algebraic numbers, unless all the coefficients $C_{1}, C_{2}, \ldots C_{m}$ are equal to zero. To prove this we establish first a lemma.

Let

$$
\alpha_{1}, \alpha_{2}, \ldots \alpha_{r}
$$

be any real or imaginary but distinct quantities, and

$$
A_{1}, A_{2}, \ldots A_{r}
$$

likewise arbitrary quantities which do not all vanish.

Similarly for the two series of quantities

Designate by

$$
\begin{gathered}
\beta_{1}, \beta_{2}, \ldots \beta_{s}, \\
B_{1}, B_{2}, \ldots B_{s} .
\end{gathered}
$$

$$
\gamma_{1}, \gamma_{2}, \ldots \gamma_{t}
$$

the distinct sums among the $r s$ sums $\alpha_{i}+\beta_{k}$ and put

$$
\begin{aligned}
A & =A_{1} e^{a_{1}}+A_{2} e^{a_{2}}+\ldots+A_{r} e^{a_{r}}, \\
B & =B_{1} e^{\beta_{1}}+B_{2} e^{\beta_{2}}+\ldots+B_{s} e^{\beta_{s}}, \\
A B & =C_{1} e^{\gamma_{1}}+C_{2} e^{\gamma_{2}}+\ldots+C_{t} e^{\gamma_{t}} .
\end{aligned}
$$

The lemma to be proved may then be stated as follows:

1. The coefficients $C_{1}, C_{2}, \ldots C_{t}$ can not all vanish.

In the proof of this theorem we may obviously assume that no one of the coefficients $A_{1}, A_{2}, \ldots A_{r}, B_{1}, B_{2}, \ldots B_{s}$ vanishes, since those that vanish may be omitted.

For brevity we shall say for the moment that of two different complex numbers $a, b, a$ is less than $b(a<b)$ when the real part of $a$ is less than the real part of $b$, or if the real parts are equal, when the imaginary part of $a$ is less than the imaginary part of $b$.

If, in this sense, $a<b, b<c$, then is $a<c$ and if $a<b$, $c<d$, then is $a+c<b+d$.

In every finite series of distinct complex numbers there is then a definite minimum and hence if $\alpha_{1}$ is the least 
among the $\alpha$ 's, $\beta_{1}$ the least among the $\beta^{\prime} s$, then is $\alpha_{1}+\beta_{1}$ the least among the $\gamma$ 's, and this sum is not equal to any other of the sums $\alpha_{i}+\beta_{k}$. Therefore $C_{1}=A_{1} B_{1}$, and $C_{1}$ is different from zero, which was to be proved.

This theorem may now be generalized by mathematical induction.

2. If

$$
\begin{aligned}
& A^{\prime}=A_{1}^{\prime} e^{a_{1}}+A_{2}{ }^{\prime} e^{a_{2}}+\ldots \\
& A^{\prime \prime}=A_{1}^{\prime \prime} e^{a_{1} \prime \prime}+A_{2} e^{a_{2} \prime}+\ldots \\
& A^{\prime \prime \prime}=A_{1}^{\prime \prime \prime} e^{a_{1} \prime \prime \prime}+A_{2}^{\prime \prime \prime} e^{a_{2} \prime \prime}+\ldots
\end{aligned}
$$

are any number of sums of the form (2), and $\gamma_{1}, \gamma_{2}, \ldots$ the distinct sums among the expressions

then in the product

$$
\alpha_{h}^{\prime}+\alpha_{i}^{\prime \prime}+\alpha_{k}^{\prime \prime \prime}+\ldots,
$$

$$
A^{\prime} A^{\prime \prime} A^{\prime \prime \prime} \ldots=C_{1} e^{\gamma_{1}}+C_{2} e^{\gamma_{2}}+\ldots
$$

not all the coefficients $C_{1}, C_{2}, \ldots$ are equal to zero.

This lemma enables us in the proof of theorem (1) to make the simplifying hypothesis that the coefficients $C_{1}$, $C_{2}, \ldots C_{m}$ are integral rational numbers.

Having assumed the existence of an equation of the form

$$
X_{1} e^{x_{1}}+X_{2} e^{x_{2}}+\ldots=0
$$

with algebraic coefficients $X_{1}, X_{2}, \ldots$ and distinct exponents $x_{1}, x_{2}, \ldots$ we may always suppose the $X_{1}, X_{2}, \ldots$ to belong to the same algebraic corpus [Körper]* $\Omega$.

* The following résumé of WEBER's definitions we take from MATHEWs's review in Nature, vol. 55 (1896), pp. 25-28: The notion of a corpus, which is of the most fundamental character, is due to Dedekind, and is as follows: Let us take a finite or infinite system of elements $\alpha, \beta, \gamma$, etc., concerning which nothing is assumed except that they can enter into rational combination according to the rules of ordinary algebra; then the totality of all rational functions of $\alpha, \beta, \gamma$, etc., except those which involve division by zero, constitutes a corpus denoted by

$$
\Omega(\alpha, \beta, \gamma, \ldots) \text {. }
$$

The simplest corpus is that of all rational numbers. This is contained in every other corpus; for if $\omega$ be any element of the corpus, then by definition the corpus contains $\omega / \omega$, that is, unity; and from this all other numbers may be derived by rational operations only.

If the elements of a corpus are all numbers it is called a numerical corpus, but the elements may be independent variables, or even variables subject to algebraical conditions.

If $\Omega(a, \beta, \gamma, \ldots)$ is any corpus, and $x$ any quantity not contained in it, 
Designate by $u_{1}, u_{2}, \ldots$ variables and construct the norm of the linear form $X_{1} u_{1}+X_{2} u_{2}+\ldots$

$$
N\left(X_{1} u_{1}+X_{2} u_{2}+\ldots\right)=\Phi\left(u_{1}, u_{2}, \ldots\right),
$$

which is an integral homogeneous function of the variables $u$ with rational coefficients, whose degree is equal to the degree of the corpus $\Omega$.

If in (6) we put

$$
u_{1}=e^{x_{1}}, u_{2}=e^{x_{2}}, \ldots,
$$

every product of the variables $u$ becomes a quantity of the form $e^{z}$, where $z$ is a sum of numbers $x$, and $\Phi\left(u_{1}, u_{2}, \ldots\right)$ becomes an expression of the form $C_{1} e^{z_{1}}+C_{2} e^{z_{2}}+\ldots$, whose coefficients are rational numbers. If the $X_{1}, X_{2}, \ldots$ do not all vanish, $C_{1}, C_{2}, \ldots$ by (2) are not all zero, even when the equal terms in the function $\Phi\left(e^{x_{1}}, e^{x_{2}}, \ldots\right)$ are combined into a single term $C e^{z}$. But if equation (5) holds, then $\Phi\left(e^{x_{1}}, e^{x_{2}}, \ldots\right)=0$ and hence

$$
C_{1} e^{z_{1}}+C_{2} e^{z_{2}}+\ldots=0 .
$$

If any of the coefficients $C_{1}, C_{2}, \ldots$ are fractions, they can be converted into integers by multiplying the equation by the lowest common denominator.

the corpus $\Omega(x, a, \beta, \gamma$,$) is said to be derived from \Omega(a, \beta, \gamma, \ldots)$ by the adjunction of $x$.

If $z$ is an undetermined variable, the polynomial .

$$
f(z)=a_{0} z^{m}+a_{1} z^{m-1}+\ldots+a_{m}
$$

is said to be a function in $\Omega$, when all the coefficients $a_{0}, a_{1}, \ldots a_{m}$ belong to $\Omega$.

When $\Omega$ is given, we may, if we like, regard all the quantities belonging to it as rational : for this reason Kronecker calls a corpus a domain of rationality.

A function in $\Omega$ is reducible (in $\Omega$ ) if it can be resolved into the product of two functions in $\Omega$. A function which is irreducible in $\Omega$ may be reducible in a corpus derived from $\Omega$ by adjunction.

Let $f(z)$ be an irreducible function in $\Omega$ of the $n^{\text {th }}$ degree in $z$; then the equation

$$
f(z)=0
$$

is assumed to have $n$ conjugate roots $z_{1}, z_{2}, \ldots z_{n}$. If $\Omega$ is a numerical corpus, the roots have actual numerical values; but this is really immaterial so far as the general algebraic theory of the corpus is concerned. By the separate adjunction of the roots, we obtain the conjugate corpora $\Omega\left(z_{1}\right)$, $\Omega\left(z_{2}\right), \ldots \Omega\left(z_{n}\right)$, each of which is called an algebraical corpus of the $n^{\text {th }}$ degree. These conjugate corpora are not necessarily all different; they may, in fact, be all identical, and the corpus is then called a Galoisian or normal corpus.

W. W. B. 
3. We need then only to show that equation (1) can not hold for any system of integral rational numbers $C_{1}, C_{2}, \ldots C_{n}$, which do not all vanish.

We assume, therefore, the existence of an equation

$$
A_{1} e^{x_{1}}+A_{2} e^{x_{2}}+\ldots=0
$$

whose coefficients $A_{1}, A_{2}, \ldots$ are integral rational numbers, not all $=0$, the exponents $x_{1}, x_{2}, \ldots$ being distinct algebraic numbers. Designate by $\Omega$ a normal corpus [Normalkörper] to which all these numbers $x_{1}, x_{2}, \ldots$ belong, and by $\theta$ a primitive number of this corpus; further by

$$
\sigma^{\prime}=\left(\theta, \theta^{\prime}\right), \sigma^{\prime \prime}=\left(\theta, \theta^{\prime \prime}\right), \ldots
$$

the substitutions of the corpus $\Omega$. If by one of these substitutions, $\sigma^{\prime}$, the numbers $x_{1}, x_{2}, \ldots$ pass into $x_{1}^{\prime}, x_{2}^{\prime}, \ldots$ then these also must be distinct.

Let now $u$ be a variable and put

$$
U=A_{1} e^{u x}{ }_{1}+A_{2} e^{u x_{2}}+\ldots
$$

Apply to this function all the substitutions $\sigma^{\prime}, \sigma^{\prime \prime}, \ldots$ and designate the functions thus obtained by $U^{\prime}, U^{\prime \prime}, \ldots$ Although we are not dealing with algebraic numbers simply, we may call the product of all these functions the norm of $U$. This product has the form

$$
N(U)=C_{1} e^{u z_{1}}+C_{2} e^{u z_{2}}+\ldots,
$$

where the $C_{1}, C_{2}, \ldots$ are likewise integral rational numbers. The quantities $z_{1}, z_{2}, \ldots$ are numbers of the corpus $\Omega$, which, if we combine terms with like exponents into a single term, we may assume to be distinct. At the same time we have on account of (7)

$$
C_{1} e^{z_{1}}+C_{2} e^{z_{2}}+\ldots=0 .
$$

The expression in the second member of (9), as shown by its formation as norm, has the property of remaining unchanged after any one of the substitutions $\sigma^{\prime}, \sigma^{\prime \prime}, \ldots$ If we expand (9) in powers of $u$, every term of the expansion must possess this invariant character; hence, if $h$ is an arbitrary positive exponent, the expression

$$
C_{1} z_{1}{ }^{n}+C_{2} z_{2}{ }^{n}+\ldots
$$

must admit the substitution; but this sum is a number of the corpus $\Omega$, and hence a rational number. This may be 
summed up as follows: If $g(z)$ designate any integral function of $z$ with rational coefficients, then is

$$
C_{1} g\left(z_{1}\right)+C_{2} g\left(z_{2}\right)+\ldots
$$

a rational number.

4. The proof of theorem I is thus reduced to the proof of the following special case: Given an equation

$$
C_{1} e^{z_{1}}+C_{2} e^{z_{2}}+\ldots+C_{m} e^{z_{m}}=0
$$

in which $z_{1}, z_{2}, \ldots z_{m}$ are distinct algebraic numbers, while $C_{1}, C_{2}, \ldots C_{m}$ and the combinations

$$
C_{1} g\left(z_{1}\right)+C_{2} g\left(z_{2}\right)+\ldots+C_{m} g\left(z_{m}\right)
$$

are rational numbers, if $g(z)$ be any integral function with rational coefficients, then must $C_{1}, C_{2}, \ldots C_{m}$ all vanish.

To prove this theorem we determine first a positive integral rational number $c$ such that

$$
x_{1}=c z_{1}, \quad x_{2}=c z_{2}, \ldots \quad x_{m}=c z_{m},
$$

become integral algebraic numbers. We assume the coefficients $C_{1}, C_{2}, \ldots C_{m}$ to be integral rational numbers and form an integral function

$$
\varphi(x)=\alpha x^{r}+a_{1} x^{r-1}+\ldots+a_{r}
$$

with integral rational numerical coefficients, which possesses the following properties :

(1) The numbers $x_{1}, x_{2}, \ldots x_{m}$ are found among the roots of $\varphi(x)=0$.

(2) The sum

$$
C_{1} \varphi^{\prime}\left(x_{1}\right)+C_{2} \varphi^{\prime}\left(x_{2}\right)+\ldots+C_{m} \varphi^{\prime}\left(x_{m}\right)=k,
$$

which by hypotheses (12), (13) is a rational integer, is different from zero.

To see that such a function as $\varphi(x)$ always exists take first a function $\chi(x)$, which satisfies only condition (1), and the second condition that no one of the numbers $x_{1}, x_{2}, \ldots x_{m}$ is a double root of $\chi(x)$. Such a function manifestly exists. We may, for example, to get a function $\chi$ take the norm of the product $\left(x-x_{1}\right)\left(x-x_{2}\right) \ldots\left(x-x_{m}\right)$ freed of any divisors that it may have in common with its derivative. Now put

$$
\varphi(x)=x^{h} \chi(x), \varphi^{\prime}\left(x_{1}\right)=x_{1}^{h} \chi^{\prime}\left(x_{1}\right), \varphi^{\prime}\left(x_{2}\right)=x_{2}^{h} \chi^{\prime}\left(x_{2}\right), \ldots
$$


Then the sum

$C_{1} \varphi^{\prime}\left(x_{1}\right)+\ldots+C_{m} \varphi^{\prime}\left(x_{m}\right)=C_{1} \chi^{\prime}\left(x_{1}\right) x_{1}{ }^{h}+\ldots+C_{m} \chi^{\prime}\left(x_{m}\right) x_{m}^{h}$ certainly cannot vanish for every exponent $h$, since otherwise, contrary to our hypothesis, $C_{1} \chi^{\prime}\left(x_{1}\right), \ldots C_{m} \chi^{\prime}\left(x_{m}\right)$ would all equal zero.

Now that the existence of a function $\varphi(x)$, such as was desired, has been established, we apply the formula $\S 205$ (12),

$$
e^{x} P(x)=F(x)+e^{\xi} Q(x) .
$$

In this we put $x=z_{1}, z_{2}, \ldots z_{m}$ and designate the absolute values of $z_{1}, z_{2}, \ldots z_{m}$ by $\xi_{1}, \xi_{2}, \ldots \xi_{m}$.

Then by (11) we obtain

$$
\begin{aligned}
0 & =C_{1} F\left(z_{1}\right)+C_{2} F\left(z_{2}\right)+\ldots+C_{m} F\left(z_{m}\right) \\
& +C_{1} e^{\xi_{1}} Q\left(z_{1}\right)+C_{2} e^{\xi_{2}} Q\left(z_{2}\right)+\ldots+C_{m} e^{\xi_{m}} F\left(z_{m}\right),
\end{aligned}
$$

and if $f(x)$ is an arbitrary integral function of the $n^{\text {th }}$ degree, we have

$$
F(z)=f(z)+f^{\prime}(z)+\ldots+f^{(n)}(z) .
$$

Designating by $p$ a natural prime number which may be made as large as we please, we put $x=c z$ [according to (13)] and

$$
f(z)=\frac{\varphi(x)^{p-1} \varphi^{\prime}(x)}{\Pi(p-1)},
$$

where $\varphi(x)$ is the function satisfying the conditions (1), (2).

Arranging in powers of $\left(x-x_{1}\right)$ we may write

$$
\begin{gathered}
\varphi(x)^{p-1} \varphi^{\prime}(x)=\varphi^{\prime}\left(x_{1}\right)^{p}\left(x-x_{1}\right)^{p-1}+A_{p}\left(x_{1}\right)\left(x-x_{1}\right)^{p} \\
+A_{p+1}\left(x_{1}\right)\left(x-x_{1}\right)^{p+1}+\ldots
\end{gathered}
$$

where $A_{p}\left(x_{1}\right), A_{p+1}\left(x_{1}\right), \ldots$ are integral algebraic numbers and rational functions of $x_{1}$. On the other hand by Taylor's theorem :

$$
f(z)=f\left(z_{1}\right)+\left(z-z_{1}\right) f^{\prime}\left(z_{1}\right)+\frac{\left(z-z_{1}\right)^{2}}{1 \cdot 2} f^{\prime \prime}\left(z_{1}\right)+\ldots
$$

and $c\left(z-z_{1}\right)=x-x_{1}$. The comparison shows that

$$
\begin{gathered}
f\left(z_{1}\right)=0, f^{\prime}\left(z_{1}\right)=0, \ldots f^{(p-2)}\left(z_{1}\right)=0, f^{(p-1)}\left(z_{1}\right)=c^{p-1} \varphi^{\prime}\left(x_{1}\right)^{p}, \\
f^{(p)}\left(z_{1}\right)=p c^{p} A_{p}\left(x_{1}\right), f^{(p+1)}\left(z_{1}\right)=p(p+1) c^{p+1} A_{p+1}\left(x_{1}\right), \ldots
\end{gathered}
$$

and hence 
$F\left(z_{1}\right)=c^{p-1} \varphi^{\prime}\left(x_{1}\right)^{p}+p c^{p} A_{p}\left(x_{1}\right)+p(p+1) c^{p+1} A_{p+1}\left(x_{1}\right)+\cdots ;$

in this $x_{1}, z_{1}$, may be replaced by $x_{2}, z_{2}$, or by $x_{3}, z_{3}$, etc.

Accordingly the integral rational number

$$
C_{1} F\left(z_{1}\right)+C_{2} F\left(z_{2}\right)+\cdots+C_{m} F\left(z_{m}\right)
$$

is congruent, to the modulus $p$, to

$$
c^{p-1}\left[C_{1} \varphi^{\prime}\left(x_{1}\right)^{p}+C_{2} \varphi^{\prime}\left(x_{2}\right)^{p}+\ldots+C_{m} \varphi^{\prime}\left(x_{m}\right)^{p}\right] .
$$

Since $C_{1}, C_{2}, \ldots, C_{p}$ are integral rational numbers, it follows that $C_{1}^{p} \equiv C_{1}, C_{2}^{p} \equiv C_{2}, \ldots(\bmod . p)$, and by an application of the polynomial theorem,

$$
\begin{aligned}
C_{1} \varphi^{\prime}\left(x_{1}\right)^{p}+C_{2} \varphi^{\prime}\left(x_{2}\right)^{p}+\ldots+C_{m} \varphi^{\prime}\left(x_{m}\right)^{p} & \equiv \\
{\left[C_{1} \varphi^{\prime}\left(x_{1}\right)+C_{2} \varphi^{\prime}\left(x_{2}\right)+\ldots+C_{m} \varphi^{\prime}\left(x_{m}\right)\right]^{p} } & \equiv k^{p}(\bmod . p) .
\end{aligned}
$$

Accordingly we have the congruence

(18) $C_{1} F\left(z_{1}\right)+C_{2} F\left(z_{2}\right)+\ldots+C_{m} F\left(z_{m}\right) \equiv c^{p-1} k^{p}(\bmod \cdot p)$.

Now the numbers $c, k$ are independent of $p$, and hence we can take $p$ so large that it will not be contained in $c$ and in $k$.

5. Hence the sum $C_{1} F\left(z_{1}\right)+C_{2} F\left(z_{2}\right)+\ldots+C_{m} F\left(z_{m}\right)$ is an integral rational number different from zero and therefore at least equal to 1.

We designate by $\varphi_{1}(x)$ the integral function derived from $\varphi(x)$ on replacing any negative coefficients among $a, a_{1}, a_{2}, \ldots$ by their positive values. The function

$$
f_{1}(z)=\frac{\varphi_{1}(x)^{p-1} \varphi_{1}^{\prime}(x)}{\Pi(p-1)}
$$

which thus results from (17) will have only positive coefficients and these coefficients are in absolute value certainly not less than the corresponding coefficients of $f(z)$, because the coefficients of $f_{1}(z)$ are derived by addition from the same numbers which in the formation of the coefficients of $f(z)$ are partly added, partly subtracted. have

Hence from formula $\S 205$ (10) letting $\xi=|x|$, we

$$
|Q(z)| \leqq \frac{\varphi_{1}(\xi)^{p-1} \varphi_{1}^{\prime}(\xi)}{\Pi(p-1)}
$$

6. And therefore for any finite value of $z Q(z)$ can be made as small as we please by a sufficient increase of $p$.

By 5 and 6 it has been shown that if $p$ be taken large enough equation (15) is impossible. Hence 4 and consequently the whole theorem I. is proved. 
This theorem now admits of manifold applications. It gives us first the transcendency of $e$, if we regard $C_{1}, C_{2}, \ldots$ $z_{1}, z_{2}, \ldots$ as integral rational numbers. It gives further the transcendency of $\pi$. For from the equation $1+e^{i \pi}=0$ it follows by $\mathrm{I}$. that $i \pi$, and consequently $\pi$, can not be algebraic.

It follows further that

For every algebraic number $x$, except $x=0, X=e^{x}$ is a transcendental number.

For every algebraic $X$, except $X=1$, every natural logarithm $x=\log X$ is a transcendental number.

For every arc which stands in an algebraically expressible relation $x$ to the radius, except $x=0, X=\sin x$ is a transcendental number.

This follows from $\mathrm{I}$, since $2 i X=e^{i x}-e^{-i x}$.

The same is true for the other trigonometric lines $\cos x$, $\tan x$, and for the chord $\frac{1}{2} \sin \frac{x}{2}$. To add one more result: The transcendental equation $\tan x=\alpha x$ for $\alpha$ algebraic has, except 0 , only transcendental numbers for roots.

\section{SHORTER NOTICES.}

A Geometrical Treatment of Curves which are Isogonal Conjugate to a Straight Line with respect to a Triangle. Part I. By I. J. Schwatr, Ph. D., University of Pennsylvania. Boston, Leach, Shewell and Sanborn, [1895]. 8vo. 6 and 46 pp. Four points serve to pair off all the points of a plane, if we take for any point its conjugate with respect to all conics through the four points. This is the reversible transformation which Durège (Ebene Curven dritter Ordnung) calls Steiner's 'Transformation. The absolute or circular points at infinity may be one of the point pairs; the conics are then rectangular hyperbolas, and the four basis points are orthocentric. Any two points $O O^{\prime}$ forming such a pair, when considered with reference to the diagonal triangle $A B C$ of the four points, are said to be isogonal conjugates, for the reason that $A O$ and $A O^{\prime}$ make opposite angles with $A B$ and $A C$.

Transformation by isogonal conjugates is thus a special view of a simple transformation of great importance. In Dr. Schwatt's pamphlet, of which a continuation is in hand, the method is applied in particular to the discussion of the transformation of a straight line, which is, of course 